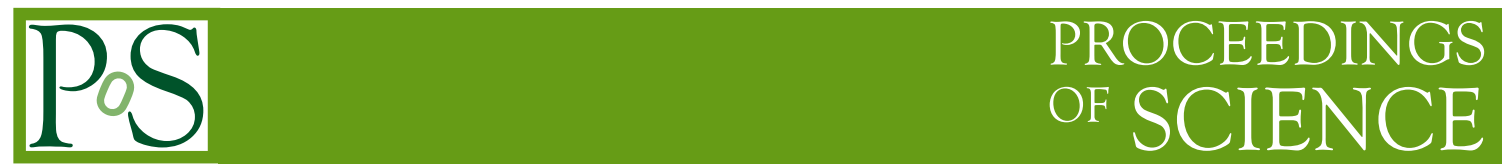

\title{
PDF uncertainties at large $x$ and gauge boson production
}

\author{
Alberto Accardi* \\ Hampton U. and Jefferson Lab \\ E-mail: accardi@jlab.org
}

I discuss how global QCD fits of parton distribution functions can make the somewhat separated fields of high-energy particle physics and lower energy hadronic and nuclear physics interact to the benefit of both. In particular, I will argue that large rapidity gauge boson production at the Tevatron and the LHC has the highest short-term potential to constrain the theoretical nuclear corrections to DIS data on deuteron targets necessary for up/down flavor separation. This in turn can considerably reduce the PDF uncertainty on cross section calculations of heavy mass particles such as $W^{\prime}$ and $Z^{\prime}$ bosons.

Xth Quark Confinement and the Hadron Spectrum

8-12 October 2012

TUM Campus Garching, Munich, Germany

\footnotetext{
* Speaker.
} 


\section{Overview}

"The coherence provided by QCD means that insights [into hadron structure] may arise from unexpected quarters. It is more than ever advisable to take a broad view that integrates across hadronic physics, and to connect with the rest of subatomic physics.” C. Quigg, 2011 [1]

The across-the-board connection I will focus on here is between the field of high-energy particle physics and lower energy hadronic and nuclear physics, and is possible thanks to the universality of the parton distribution functions (PDFs) of the proton, which allows one to calculate a variety of processes from a common set of quark and gluon momentum distributions. These processes range from high-energy physics interactions such as in $\bar{p}+p$ collisions at the Tevatron or $p+p$ collisions at the Large Hadron Collider (LHC) to lower energy hadronic physics interactions on proton as well as nuclear targets. The former are designed to study QCD and electroweak interactions at the parton level, as well as to search for the Higgs boson and for physics beyond the standard model. The latter are designed to explore how QCD builds a hadron out of 3 valence quarks, how its quantum numbers are built up from these and from the "sea" of quark-antiquark pairs and gluons, how its properties change in a nuclear medium, and how a nucleus as a bound state of protons and neutrons emerges from the underlying microscopic quark and gluon degrees of freedom.

One powerful tool that exploits the PDF universality are the so called "global PDF fits". Their original raîson d'être is to utilize experimental data from a number of processes, combined with perturbative QCD calculations of the relevant parton-parton cross sections, in order to extract the non perturbatively calculable parton distributions. These in turn can be used to calculate processes not included in the fits, for example the expected rates of Higgs boson production in various channels, or the QCD background to various standard or beyond-the-standard model processes, such as production of $W^{\prime}$ and $Z^{\prime}$ gauge boson, Kaluza-Klein resonances, and so on. In this sense, increasing the number of data points to be fitted by including more processes, and improving the theoretical calculations to include a larger portion of the available kinematics, is extremely useful to reduce the uncertainties in the extracted PDFs, thus yielding more accurate predictions or background calculations. This is one way in which lower energy hadronic and nuclear data, which typically access lower momentum scales and larger parton momentum fractions $x$ inside a proton than at colliders, can improve the study of high-energy processes.

However, as I will argue here, this phenomenologically very important connection is not necessarily the most far reaching. For example, data on $W$ and $Z$ forward rapidity boson production at Tevatron and the LHC, which can reach large values of $x$, can constrain the extrapolation to $x=1$ of the down-quark to up-quark ratio in the proton, and (with enough statistical precision) indicate which nonperturbative proton structure model best captures the effects of confinement on hadron structure. Here we have collider physics providing insights on hadronic physics.

Likewise, but less obviously, global PDF fits can be used as a tool to study the structure of the nucleus and the differences between bound and free protons and neutrons, for which current models display large theoretical uncertainties. This is possible, e.g., by exploiting the interplay of Deep Inelastic Scattering (DIS) data in deuteron targets, which allow one to extract a nuclear model dependent $d$ quark distribution at large $x$ [2-4], and large rapidity weak interaction processes on 


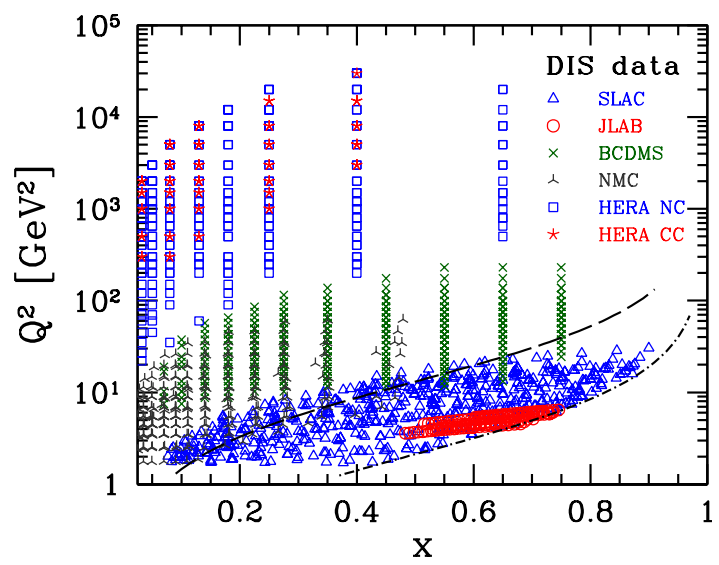

Figure 1: Kinematic coverage in Bjorken $x_{B}$ and $Q^{2}$ of the DIS data used in the CJ12 fits [4]. The $W^{2} \gtrsim 14 \mathrm{GeV}^{2}$ cut typically use in global PDF fits and the $W^{2}>3$ $\mathrm{GeV}^{2}$ cut used in the CJ12 analysis are indicated by the dashed and dot-dashed lines, respectively.

proton targets, which also depend on the $d$ quark but are naturally free from nuclear effects. Since an unrealistic nuclear correction would pull the $d$ quark extracted from a global fit away from that required by the deuteron target data, constraints on the viable nuclear models can then be obtained by studying tensions in these two data sets under global fits. Global fits can thus turn detectors such as CDF at the Tevatron or LHCb at the LHC into nuclear physics experiments complementing more traditional lower energy ones on fixed nuclear targets, such as at Jefferson Lab [5, 6].

It is the goal of this talk to substantiate these claims.

\section{The CTEQ-Jefferson Lab global PDF fits}

Plentiful DIS data exist from fixed-target experiments at large $x$ and low scale $Q^{2}$, which is typically excluded in global QCD fits by imposing cuts on the invariant mass $W \gtrsim 3.8 \mathrm{GeV}$ in order to exclude the region where higher-twists and other subleading in $1 / Q^{2}$ effects are important, see Fig. 2. The CTEQ-Jefferson Lab (CJ) collaboration [7] was formed with the initial aim to include these subleading effects in the perturbative calculations, in particular target mass corrections and parametrized higher-twist terms, and to investigate the effect of relaxing the cut to just inside the resonance region, $W>1.7 \mathrm{GeV}$. The resulting fits [2] were stable with the weaker cuts, and the larger database led to significantly reduced errors, in particular up to 40-60\% on the $d$ quark at large $x \gtrsim 0.6$, where precise data sensitive to this distribution are otherwise scarce. As the $d$ quark flavor separation at large $x$ is almost entirely dependent on DIS on deuteron targets, corrections for nuclear Fermi motion and binding effects were included by convoluting the nucleon structure functions with a smearing function calculated from the deuteron wave function, and found to be non negligible also in the "safe" region defined by the larger $W$ cut discussed above.

A subsequent analysis, referred to as CJ11 [3], explored in detail the theoretical uncertainties arising at large $x$, and a public PDF release including PDF errors and valid in the whole $10^{-5} \lesssim$ $x \lesssim 0.9$ range was provided in Ref. [4], that I will refer to as CJ12.

In these two papers, a more flexible valence $d_{v}$ quark parametrization at large- $x$ with a small admixture of the valence $u_{v}$ PDF was used,

$$
d_{v}(x) \rightarrow a_{0}^{d_{v}}\left[d_{v}(x) / a_{0}^{d_{v}}+b x^{c} u_{v}(x)\right]
$$

with $b$ and $c$ as two additional parameters. The result of this modification is that $d_{v} / u_{v} \rightarrow a_{0}^{d_{v}} b$ as $x \rightarrow 1$, provided $d_{v}$ goes faster to 0 than $u_{v}$, which is usually the case. A finite, nonzero value of 


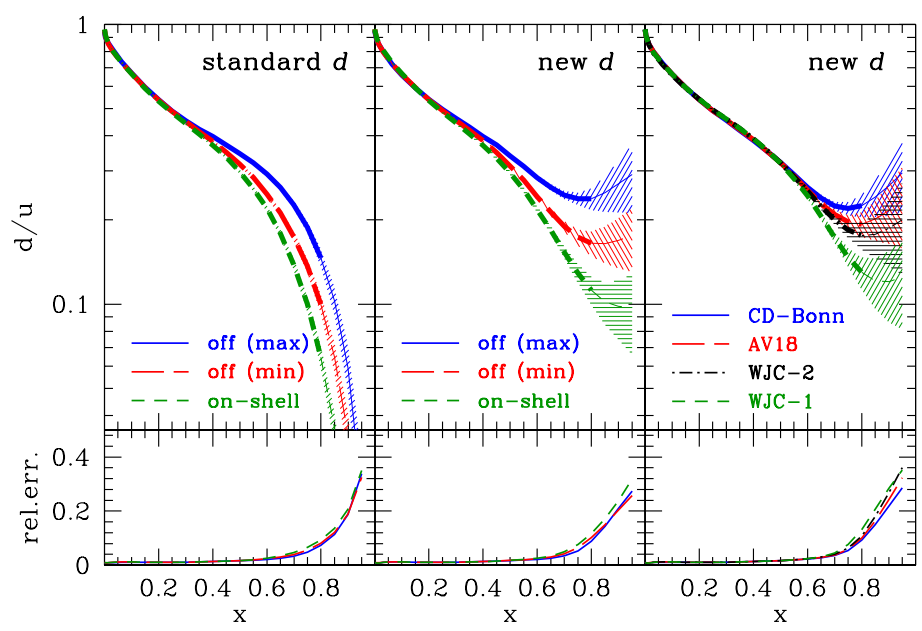

Figure 2: The CJ11 $d / u$ ratio at $Q^{2}=10 \mathrm{GeV}^{2}$ obtained with different $d$ quark parametrization and nuclear corrections: (left) standard $d$ quark [2] and AV18 deuteron wave function for several off-shell corrections models; (middle) modified $d$ quark parametrization; (right) dependence on the wave function for a fixed offshell correction (mKP). The shaded bands and bottom panels show the $\Delta \chi=1$ absolute and relative PDF errors, respectively. For details, see Ref. [3].

this ratio is indeed expected in several nonperturbative models of hadron structure, see Ref. [8]. It is also required from a purely practical point of view, as it avoids potentially large biases on the $d$ quark PDF central value [3], as illustrated in Fig. 2, as well as on its PDF error estimate [4].

In a global fit, it is the combination of PDFs and nuclear corrections which is constrained by deuterium data, not the PDFs alone. At large $x$, the $u$ quark is well constrained by free proton DIS and other data, so that when fitting the corrected deuterium data the $d$ quark will be the distribution most sensitive to the nuclear corrections. Correspondingly, this acquires a significant theoretical uncertainty due to the modeling of nuclear corrections for DIS on deuterium targets, which I refer to as "nuclear uncertainty" in short. This was evaluated by considering nuclear corrections ranging from mild, corresponding to the hardest of the modern deuteron wave functions (WJC-1) coupled to a $0.3 \%$ nucleon off-shellness, to strong, corresponding to the softest of the wave functions (CDBonn) and a large, $2.1 \%$ nucleon off-shellness, with a central fit corresponding to the AV18 wave function and a $1.2 \%$ nucleon off-shellness. The resulting PDFs are labeled CJ12min, CJ12max, and CJ12mid, respectively.

The uncertainties in the CJ12 $d$ quark distribution are illustrated in Fig. 3 left. The red band is the PDF error on the central fit induced by the experimental uncertainty in the fitted data, and calculated with the Hessian method and a tolerance factor $T=10$. The blue band represents the nuclear uncertainty, obtained as an envelope of the CJ12min and CJ12max fits, and is of the same order of magnitude as the PDF error. The green band is the PDF error in a fit without the deuterium data, and exceeds the band which includes the nuclear uncertainties. This clearly demonstrates the usefulness of the deuterium DIS data, even in the presence of the nuclear uncertainties that its use introduces.

\section{Applications}

I will discuss in turn a hadronic physics and a collider physics application of the fitted CJ12 PDFs, and explore the consequences of the nuclear uncertainties in either case.

The ratios of the $d$ to $u$ PDFs for the three CJ12 sets are shown in Fig. 3 right. These are constrained up to $x \approx 0.8$ by the enlarged data set considered in the CJ fits, but can be confidently extrapolated to $x=1$ thanks to the modified $d$ quark parametrization (2.1). As the magnitude of 

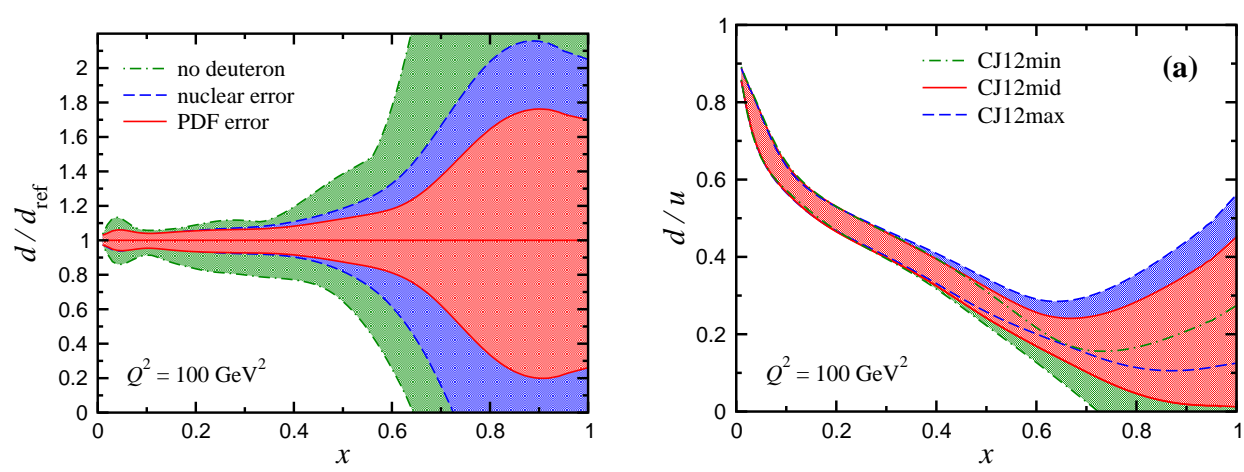

Figure 3: Left: PDF uncertainties for the CJ12mid $d$ quark PDFs (solid) compared with the total uncertainty including nuclear corrections (dashed) and with a fit excluding all deuterium data (dot-dashed), relative to the reference CJ12mid set at $Q^{2}=100 \mathrm{GeV}^{2}$. Right: $d / u$ ratio at the same $Q^{2}$ for the CJ12min (dot-dashed), CJ12mid (solid) and CJ12max (dashed) PDFs. Plots taken from Ref. [4].

the nuclear corrections increases, the $d / u$ intercept at $x=1$ rises as discussed in detail in Ref. [4]. Including the PDF errors, it was found that $d / u \underset{x \rightarrow 1}{\longrightarrow} 0.22 \pm 0.20$ (PDF) \pm 0.10 (nucl), where the first error is from the PDF fits and the second is from the nuclear correction models. These values encompass the 0-0.5 range of available theoretical predictions [8]. However, it is also clear that a relatively modest improvement in statistical precision and reduction of nuclear uncertainty would allow one to restrict the range of allowable physical mechanisms.

The large nuclear uncertainty in the quark PDFs (and that in the gluon PDF arising from its jet data induced anticorrelation with the $d$ quark [3]) have also potentially profound implications for future collider experiments. To illustrate this in general terms, consider the differential parton luminosities for production of an object of mass $\sqrt{\hat{s}}$ at rapidity $y$ in a hadronic collision of center of mass energy $\sqrt{s}, d L_{i j} / d y=\left[f_{i}\left(x_{1}, \hat{s}\right) f_{j}\left(x_{2}, \hat{s}\right)+(i \leftrightarrow j)\right] /\left[s\left(1+\delta_{i j}\right)\right]$, where $x_{1,2}=\tau e^{ \pm y}$, with $\tau=\sqrt{\hat{s} / s}$. The differential luminosities, normalized to the reference fit calculation, are shown in Fig. 4 as a function of $\tau$ for three values of rapidity (since the ratios are largely independent of $Q^{2}$, these plots are also independent of $s$ ). The sensitivity to large $x$ PDFs, hence to nuclear uncertainties, grows the larger the mass of the produced object and the larger its rapidity at a given mass. For example, the nuclear uncertainty becomes relevant for $W$ production at rapidity larger than 2 at the Tevatron, and larger than 3.5 at the LHC [9], as we shall see in more detail. For particles of heavier mass, such as $W^{\prime}$ and $Z^{\prime}$ bosons, the nuclear uncertainty at large $x$ may become large also in the inclusive production cross section [9], as illustrated in Figure 3. As the current LHC limits put the $W^{\prime}$ and $Z^{\prime}$ masses approximately above $2.6 \mathrm{GeV}$ for Standard-Model like couplings [10], one can appreciate how nuclear uncertainties and other large- $x$ theoretical uncertainties may significantly affect the interpretation of signals of new particles and an accurate measurement of their properties, which require a precise determination of QCD backgrounds.

\section{Constraining the nuclear corrections}

Given the current size of nuclear uncertainties, and their impact on phenomenology across low-energy hadron physics and high-energy particle physics, it is imperative to reduce this source of theoretical uncertainty. 


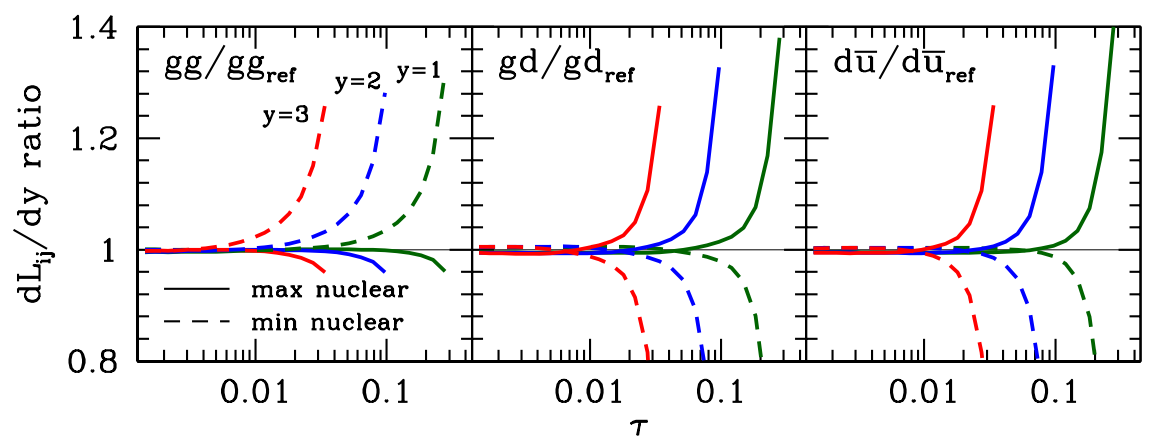

Figure 4: CJ11 differential parton luminosities for $g g, g d$ and $d \bar{u}$ parton collisions at fixed rapidity $y=1,2$ and 3, as a function of $\tau=\sqrt{\hat{s} / s}$, illustrating the variations due to the choice of nuclear corrections [3].

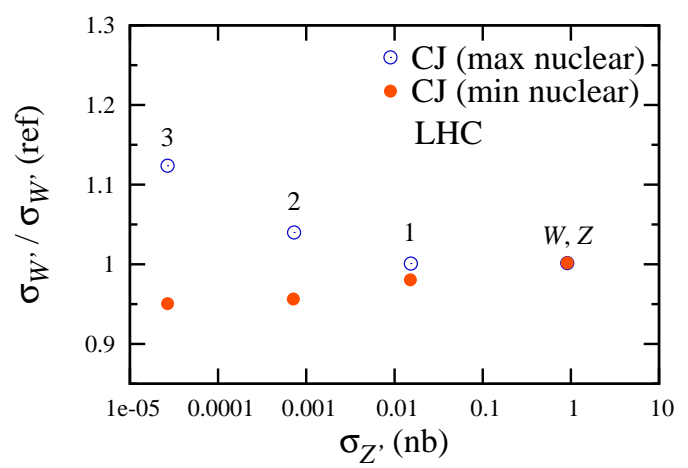

Figure 5: Ratio of $W^{\prime}$ boson $\sigma_{W^{\prime}}=\sigma_{W^{\prime+}}+\sigma_{W^{\prime}}$ cross section, versus the $Z^{\prime}$ boson cross section $\sigma_{Z^{\prime}}$ for varying $Z^{\prime}$ boson masses (in $\mathrm{TeV}$ next to the points). The cross sections are computed for $\sqrt{s}=7 \mathrm{GeV}$ from CJ11 PDFs [3] with minimum (filled red circle) and maximum (open blue circle) nuclear corrections, relative to the reference cross section calculated from the central CJ11 PDFs. Plot taken from Ref. [9].

On the theory side the main difficulty resides in the interplay of the hard interaction at the nucleon level, described in terms of partonic degrees of freedom and calculable directly from the QCD Lagrangian, and the comparatively soft nucleon-nucleon interactions described in terms of hadronic degrees of freedom with effective interactions fitted to nucleon elastic scattering data. Oftentimes the soft nuclear and hard nucleonic interactions are assumed to factorize, even though this is not formally guaranteed the way QCD factorization theorems allow to separate partonic interactions from nonperturbative PDFs. Moreover, the medium modifications of the nucleons are almost unknown experimentally, especially in the nucleon momentum range relevant for inclusive DIS. Therefore, theoretical models of nuclear corrections seem at present irreducibly unconstrained, and comparison to non-inclusive DIS experimental data is mandatory for further theoretical progress.

If, however, one's purpose was solely to obtain a precise enough but nuclear uncertainty free extraction of the $d$ quark, one would need new experimental data to compensate for the loss in statistics deriving from excluding the deuteron data shown in Figure 3 left. This new data can come from 2 sources (for references, see [4]). The first is data on nuclear targets, but with observables designed to minimize the impact of nuclear corrections, for example, DIS on deuteron with detection of a slow spectator proton, which guarantees that the electron scatters on a quasi-free neutron, or inclusive DIS on 3-Helium and Tritium, where the nuclear effects largely cancel in the ratio of the respective cross sections (future BONUS 12 and MARATHON experiments at Jefferson Lab, respectively). The second source is weak interactions in proton target collisions. These contribute, through photon- $Z$ boson interference, to electron and positron DIS cross sections at large $Q^{2}$ only (for which, unfortunately, the combined run-I HERA data have insufficient large $x$ coverage), but 

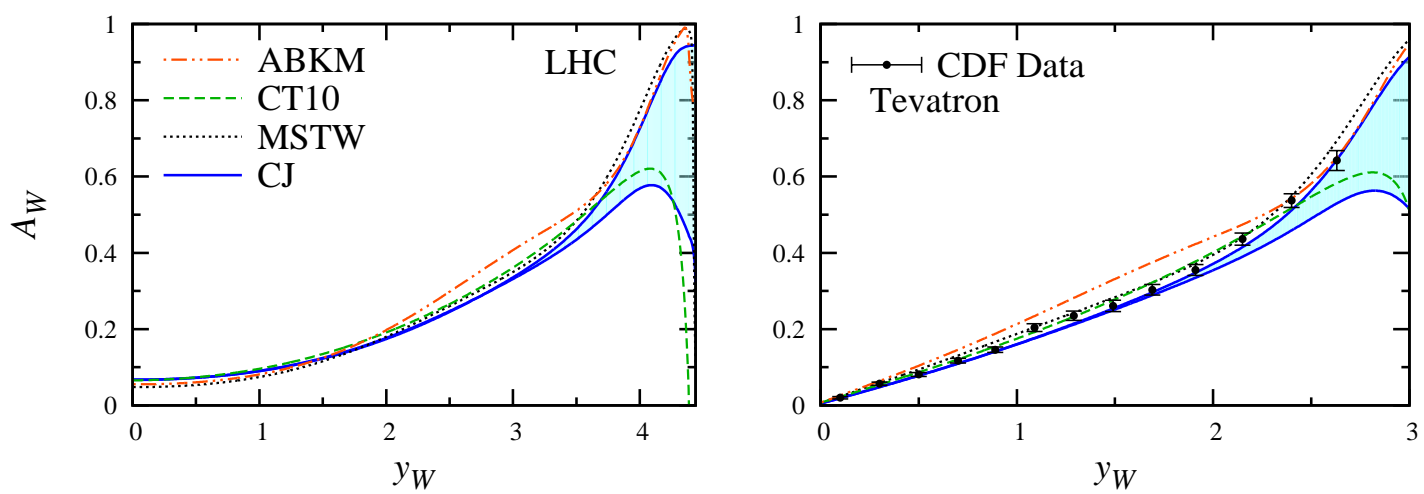

Figure 6: $W$ boson asymmetry $A_{W}$ as a function of the $W$ rapidity $y_{W}$ at LHC and Tevatron, computed from CJ11 PDFs with minimum (upper blue solid) and maximum (lower blue solid) nuclear corrections. The asymmetries using the ABKM, CT10 and MSTW PDF sets are also shown. Plot taken from Ref. [9].

are of leading order in parity violating charge asymmetries (future SOLID and PVDIS experiments at Jefferson Lab). In principle, weak charged currents can also be directly measured in neutrino and anti-neutrino scattering on proton target, for which however the old WA21/22 data cannot be easily used in global fits, and no new data is planned for the foreseeable future. This leaves one with $W$ and $Z$ boson production in $p+\bar{p}$ and $p+p$ collisions at Tevatron and the LHC, which I shall focus on next.

As discussed at the end of Section 1, global QCD fits allow for the intriguing possibility to combine deuteron and proton target data to obtain experimental constraints on the nuclear models, and at the same time fully utilize the available statistics of the nuclear target data. This is very well demonstrated by considering the directly reconstructed $W$ asymmetry at Tevatron. As shown in Figure 6, this process is very sensitive to nuclear corrections at $y \gtrsim 2$, and the comparison with the very precise CDF data [11], as well as the total $\chi^{2}$ values in the CJ12 fits, suggests that nuclear effects are somewhere between the minimum and central ones considered in the CJ12min and CJ12mid fits, respectively, as also confirmed by a similar analysis presented in Ref. [12]. On the phenomenological side, this result disfavors nonperturbative proton models based on the SU(6) spin-flavor symmetry, which predicts $d / u \rightarrow 1 / 2$ as $x \rightarrow 1$. More importantly, it exemplifies the power of global fits in combining data from across the board, and is the first step in establishing the experimental foundation that was until now missing for a qualitative jump in our ability to theoretically understand and describe high-energy processes in nuclei - and this without even utilizing nuclear targets!

The $W$ charge asymmetry at CDF unfortunately seems the only observable that currently has this potential. The lepton asymmetry has insufficient large $x$ coverage due to decay vertex smearing, while $Z$ rapidity distributions from $C D F$ and $D \varnothing$ have insufficient precision [4]. At the LHC one would need measurements with better than $10 \%$ precision at $y \gtrsim 3.5$, namely, at the edge of the LHCb acceptance for $\sqrt{s}=7 \mathrm{TeV}$, and the experimental accuracy of the $W$ asymmetry is inherently reduced due to the difference between $p+p$ scattering compared to $p+\bar{p}$ [9]. Nonetheless, inclusion of the available data in the CJ fits is underway to quantify its impact on nuclear uncertainty constraints. The potential of large rapidity measurements in proton-proton collisions at the upgraded RHIC detectors, or the proposed AFTER@LHC experiment, should also be explored. 


\section{Conclusion}

The recent CTEQ-Jefferson Lab collaboration investigations, culminating in the public CJ12 PDF realease [4], have demonstrated the intimate interconnection of hadronic and high-energy physics, exemplifying one of the across-the-board connections called for in the opening quote by C. Quigg for further progress in these fields. Namely, global QCD fits have become capable of constraining theoretical models of nuclear corrections in the deuteron. Not only will this reduce the nuclear uncertainty on the fitted PDFs with important phenomenological consequences on physics ranging from nonperturbative proton structure to beyond the standard model interactions, it will also provide a new avenue for progress in the theoretical understanding of high-energy processes involving nuclei, using weak interactions on proton targets from Jefferson Lab to the LHC.

Acknowledgments: I am very grateful to my CJ colleagues for the enjoyable and productive collaboration, and to the organizers for their kind support. This work was supported by the DOE contract No. DE-AC05-06OR23177, under which Jefferson Science Associates, LLC operates Jefferson Lab, and by the DOE contract DE-SC008791 and NSF award No. 1002644.

\section{References}

[1] C. Quigg, The Future of Hadrons: The Nexus of Subatomic Physics, in proceedings of HADRON 2011, 13-17 June 2011, Munich, Germany [arXiv:1109.5814 [hep-ph]].

[2] A. Accardi et al., New parton distributions from large- $x$ and low- $Q^{2}$ data, Phys. Rev. D 81 (2010) 034016 [arXiv:0911.2254 [hep-ph]].

[3] A. Accardi et al., Uncertainties in determining parton distributions at large $x$, Phys. Rev. D 84 (2011) 014008 [arXiv:1102.3686 [hep-ph]].

[4] J. F. Owens, A. Accardi and W. Melnitchouk, Global parton distributions with nuclear and finite- $Q^{2}$ corrections, arXiv:1212.1702 [hep-ph].

[5] D. Higinbotham, W. Melnitchouk, A. W. Thomas (Eds.), New Insights into the Structure of Matter: The First Decade of Science at Jefferson Lab, J. Phys. Conf. Ser. 299 (2011).

[6] J. Dudek et al., Physics Opportunities with the 12 GeV Upgrade at Jefferson Lab, Eur. Phys. J. A 48 (2012) 187 [arXiv:1208.1244 [hep-ex]].

[7] The CTEQ-Jefferson Lab (CJ) collaboration website, http://www.jlab.org/cj.

[8] R. J. Holt and C. D. Roberts, Distribution Functions of the Nucleon and Pion in the Valence Region, Rev. Mod. Phys. 82 (2010) 2991 [arXiv:1002.4666 [nucl-th]].

[9] L. T. Brady, A. Accardi, W. Melnitchouk and J. F. Owens, Impact of PDF uncertainties at large $x$ on heavy boson production, JHEP 06 (2012) 019 [arXiv: 1110.5398 [hep-ph]].

[10] G. Dissertori, Review of LHC results on proton-proton physics: Present highlights and future, these proceedings.

[11] T. Aaltonen et al., Direct Measurement of the $W$ Production Charge Asymmetry in p $\bar{p}$ Collisions at $\sqrt{s}=1.96 \mathrm{TeV}$, Phys. Rev. Lett. 102 (2009) 181801 [arXiv:0901.2169 [hep-ex]].

[12] A. D. Martin et al., Extended Parameterisations for MSTW PDFs and their effect on Lepton Charge Asymmetry from W Decays, arXiv:1211.1215 [hep-ph]. 